\title{
COMPUTATIONAL APPROACH TO HYPERELLIPTIC RIEMANN SURFACES
}

\author{
J. FRAUENDIENER AND C. KLEIN
}

\begin{abstract}
We present a computational approach to general hyperelliptic Riemann surfaces in Weierstrass normal form. The surface is either given by a list of the branch points, the coefficients of the defining polynomial or a system of cuts for the curve. A canonical basis of the homology is introduced algorithmically for this curve. The periods of the holomorphic differentials and the Abel map are computed with the Clenshaw-Curtis method in order to achieve spectral accuracy. The code can handle almost degenerate Riemann surfaces. This work generalizes previous work on real hyperelliptic surfaces with prescribed cuts to arbitrary hyperelliptic surfaces. As an example, solutions to the sine-Gordon equation in terms of multi-dimensional theta functions are studied, also in the solitonic limit of these solutions.
\end{abstract}

\section{INTRODUCTION}

Among compact Riemann surfaces of higher genus, hyperelliptic surfaces have clearly the most applications and can be seen as straight forward generalizations of elliptic surfaces. It can be shown for instance that all Riemann surfaces of genus 2 are hyperelliptic. For this reason alone and for their relative simplicity, hyperelliptic Riemann surfaces are important examples in algebraic geometry. Their first applications in mathematical physics were Neumann's 31 integration of the motion of a rigid body on a surface and the Kowalewskaja top 28. In the 1970s, quasiperiodic solutions of the celebrated Korteweg-de Vries (KdV) equation were given by Its and Matveev in terms of multidimensional theta functions associated to hyperelliptic Riemann surfaces, see [1] and 9] for an account of the history. It could be shown that many $1+1$ dimensional completely integrable equations such as KdV have finite gap solutions defined on hyperelliptic surfaces, see for instance [1] and references therein for the nonlinear Schrödinger (NLS) and 27 for the sine-Gordon (SG) equation, 23] and 24] for the Ernst equation and [20] and references therein for the Camassa-Holm equation. Hyperelliptic Riemann surfaces also appear in the asymptotic description of dispersive shocks, i.e., highly oscillatory regions in the solution for these 1+1-dimensional equations, see e.g. [17, 18, 21, 29 for KdV and NLS. In this paper, we present an efficient numerical approach to general hyperelliptic Riemann surfaces even in almost degenerate situations.

As all compact Riemann surfaces, hyperelliptic surfaces can be defined via algebraic curves which, in the present case, can be written in Weierstrass normal form

$$
\mu^{2}=\left(z-z_{1}\right)\left(z-z_{2}\right) \ldots\left(z-z_{N}\right)
$$

where $(\mu, z) \in \mathbb{C}^{2}$ and where $z_{i} \in \mathbb{C}$ (all $z_{i}$ distinct) for $i=1, \ldots, N$. For $N=1,2$, the surface has genus $g=0$, for $N=3,4$, it is elliptic $(g=1)$, for $N>4$ the surface

Date: August 12, 2014

Key words and phrases. hyperelliptic Riemann surfaces, Abel map, spectral methods, sineGordon equation.

This work has been supported in part by the Marie-Curie IRSES project RIMMP and the Royal Society of New Zealand. 
is hyperelliptic and has the genus $g=N / 2-1$ for $N$ even and $g=(N-1) / 2$ for $N$ odd. Hyperelliptic surfaces in Weierstrass form can thus be represented in a standard way as two-sheeted coverings of the complex plane which are branched at the zeros $z_{i}, i=1, \ldots, N$ of the polynomial on the right hand side of (1), the branch points of the surface. A general point on the two-sheeted covering is denoted by $(z, \mu)$. The hyperelliptic involution $\sigma$ interchanges the sheets, $\sigma(z, \mu)=(z,-\mu)$. The sheets of the covering will be denoted in the following as + and - sheet where the sign of $\mu$ is defined at some base point $z_{B}$ with $\mu\left(z_{B}\right) \neq 0$. In the case of odd $N$, the surface is branched at infinity, otherwise all branch points are finite. Note that surfaces obtained from (1) via birational transformations of the form $(\mu, z) \rightarrow$ $\left(R_{1}(\mu, z), R_{2}(\mu, z)\right)$, where $R_{1}(\mu, z)$ and $R_{2}(\mu, z)$ are rational in both arguments, i.e., by replacing $\mu$ and $z$ in (1) by $R_{1}(\mu, z)$ respectively $R_{2}(\mu, z)$, obviously also represent a hyperelliptic algebraic curve. We will here only discuss curves in the Weierstrass normal form (1) (for a discussion of how to check whether a given algebraic curve is hyperelliptic and how to find the transformation to Weierstrass form, see for instance the appendix of 7$]$ ).

The many applications of hyperelliptic curves make efficient numerical procedures necessary. Different approaches are known: treating the hyperelliptic curves as special cases of general algebraic curves, see [5, 6] and [16]; uniformization techniques as described in 3, 26]; and in terms of theta functions [10. Hyperelliptic curves can be treated simply as special cases of general algebraic curves, for instance with the approach in 16. However, the hyperellipticity implies two important simplifications which make special approaches for hyperelliptic curves much more efficient: firstly, it is not necessary to solve an algebraic equation in order to analytically continue the roots $\mu$ in (1) which, as was shown in [16], takes almost $80 \%$ of the computing time - it is just a square root here. This can be efficiently computed for several values at the same time, and the analytic continuation is simply the removal of inappropriate sign changes on a system of algorithmically identified cuts on the surface. Also, the homology basis can be fixed a priori. Secondly, the holomorphic differentials are known a priori for hyperelliptic surfaces (see for instance 1]): the one-forms

$$
\nu_{k}=\frac{z^{k-1} d z}{\mu(z)}, \quad k=1, \ldots, g
$$

form a basis of the space of holomorphic one-forms on the surface. Thus, the differentials do not need to be determined by the algorithm. This means that the operations, which are most time consuming in the case of general algebraic curves, become either almost trivial or unnecessary in the hyperelliptic case. Therefore, efficient numerical approaches for hyperelliptic curves are almost 100 times faster than more general codes. Hence, given the importance of the hyperelliptic case, it is well worth to consider optimized adapted approaches.

In 13 15, we have presented a computational approach to real Riemann surfaces with spectral convergence, i.e., an exponential decrease of the numerical error with the numerical resolution. This approach allowed us to study modular properties of hyperelliptic functions. Loosely speaking, this is the dependence of functions defined on a family of hyperelliptic surfaces on the branch points $z_{i}$ of the defining equation (1), see e.g. 22] and references therein. This is also important in the context of solutions to the Ernst equation found by Korotkin 24, see 23 for a review, where branch points of the underlying surface are parametrized by the physical coordinates. The codes in 14, 15] are also able to address the case where branch points almost coincide, a case known as the solitonic limit in the context of quasiperiodic solutions in terms of theta functions to integrable equations (in this limit, the solutions are no longer periodic, but localized multi-solitons). Since the 
signs of the square root in these codes were only fixed locally, they were limited to particular real Riemann surfaces, i.e., branch points that were either all real or all pairwise complex conjugate. In addition, the branch points had to be known $a$ priori and be arranged in pairs corresponding to the cuts of the surface.

In the present paper, we present a robust implementation of the square root which allows the treatment of general hyperelliptic curves. In addition, an algorithm is given for selecting a basis of the homology for a given (unordered) list of branch points. The curve (1) can be given in one of 3 forms: i) as an algebraic equation in the form

$$
\mu^{2}=a_{N} z^{N}+a_{N-1} z^{N-1}+\ldots+a_{0}
$$

with $a_{i}, i=0, \ldots, N$ some complex constants, and the branch points are determined numerically, ii) in the form (1), and iii) in terms of the branch points of the curve (1) given as in 14,15 in pairs. In the first two cases, we present an algorithm to obtain a system of cuts for the surface. The only condition is that the branch points are sufficiently separated (as detailed in the following sections). In the third case, they can almost coincide which allows for the study of almost solitonic situations.

As an example we will study quasiperiodic solutions to the SG equation

$$
u_{\xi \eta}=4 \sin (u)
$$

where $\xi, \eta, u$ are real, and where the index denotes a partial derivative. The SG equation has been first discovered in the theory of surfaces as the Gauss-Codazzi equation for surfaces of constant negative curvature, see 2, 4] or [23, 25] for a more modern presentation. Note that the complete integrability of the SG equation was already shown by Bianchi 2 who managed to introduce a spectral parameter into the Gauss-Weingarten system for the surface, which plays the role of a Lax pair. As illustrated for instance in 23 , the surfaces with constant negative curvature corresponding to SG solitons include the pseudosphere and the helicoid. The SG equation was also studied as a quantum field theoretical model in 11].

The paper is organized as follows: In section 2, an algorithm is presented to establish a system of cuts for a given list of branch points $z_{i}, i=1, \ldots, N$. In section 3 , the square root $\mu$ in $(1)$ is numerically computed and analytically continued along these cuts. Also, the periods of the holomorphic differentials and the Abel map are computed with the Clenshaw-Curtis method. In section 4, we study solutions to the SG equation in terms of multidimensional theta functions on hyperelliptic curves. We add some concluding remarks in section 5 .

\section{Construction of a Basis of the homology}

In this section we explain how to construct a basis of the homology of the surface if the hyperelliptic curve is given in the form (1) or (3), i.e., essentially for a list of the branch points $z_{i}, i=1, \ldots, N$ in (1).

2.1. Computation of the branch points. If the hyperelliptic curve is given in the form (3), i.e., via the coefficients $a_{i}, i=0, \ldots, N$ of the polynomial in $z$, then the branch points are exactly the points where $\mu$ vanishes, which means the zeros of the polynomial. A standard way to compute the zeros of a polynomial numerically is to construct the companion matrix, a matrix which has this polynomial as its characteristic polynomial. The eigenvalues of this matrix are by definition the zeros of the original polynomial. In Matlab, this approach is used via the command roots. Note that the eigenvalues are computed with machine precision $\left(10^{-16}\right.$ in our case, but in practice limited to $\approx 10^{-14}$ due to rounding errors), but this does not imply that the zeros of the polynomial are obtained with machine precision. Problems arise if the zeros are too close to each other or of higher multiplicity because of the 
finite precision used in the computations, see for instance [35] and references given therein for more details.

To avoid such problems, we always assume that the problem is well conditioned, i.e., that all zeros have multiplicity 1 and are well enough separated. To characterize the latter condition we consider the quotient $\Delta$ of the largest distance between any two finite branch points divided by the smallest distance between any two branch points,

$$
\Delta:=\frac{\max _{i, j=1, \ldots, N}\left|z_{i}-z_{j}\right|}{\min _{i, j=1, \ldots, N ; i \neq j}\left|z_{i}-z_{j}\right|},
$$

and require that $\Delta$ is always smaller than $10^{6}$. In this case the Matlab command roots produces a list of the zeros of the polynomial and thus the branch points. If considerably higher values of $\Delta$ are to be treated, the branch points will be determined with less and less accuracy. To obtain reliable results in this case, the branch points have to be given in pairs as discussed below.

2.2. Identifications of cuts and basis of the homology. Thus, whether the curve is given in the form (1) or (3), we essentially start in both cases with the set of branch points $z_{i}$. To define a system of cuts between them, the branch points need to be sorted. The general idea is as follows: We define a function $f: \mathbb{C} \rightarrow \mathbb{R}$ which separates the branch points, and then we sort them according to the values $f\left(z_{i}\right)$. This can be done in several ways. Here, we restrict ourselves to the following method: We determine a central point $c$ taken as the arithmetic mean of the finite branch points $z_{i}, i=1, \ldots, N$. To reduce the possibility of degeneracies, we add a random complex number of the order of machine precision to $c$. Then we compute the argument of the lines $z_{i}-c$, i.e., we put $f(z)=\arg (z-c)$. We order the branch points in increasing order of $f\left(z_{i}\right)$ into a sequence $\left(z_{i}\right)$ (in case of degeneracies, the corresponding points are ordered according to their modulus, but highly symmetric versions can be conveniently treated with the third approach detailed below). Then we define the cuts $C_{j}$ to be between the branch points $z_{2 j+1}$ and $z_{2 j+2}$ for $j=0, \ldots, g$, i.e., $C_{0}=\overline{z_{1} z_{2}}$ etc. A general picture is given in Fig. 1 . Note, that the cuts tend to form a line curving towards the left. If $N$ is odd, the surface is branched at infinity, and we add $\infty$ as the first branch point by following the line through $c$ with the argument of $z_{1}-c$ to infinity. This defines the first cut in this case.

To obtain a basis of the homology, the closed cycles are chosen according to the cuts in Fig. 1 as can be seen in Fig. 2 The cycles denoted by $a_{0}, a_{1}, \ldots a_{g}$, enclose the cuts $C_{j}$, circling around them exactly once in clock-wise orientation. We use $a_{1}, \ldots, a_{g}$ as the so-called $a$-cycles. Note that $a_{0}$ is homologous to the negative sum of the $a_{i}, i=1, \ldots, g$ and thus not part of the canonical basis of the homology used here. But it has a role in the ensuing computations and is, therefore, already mentioned at this point. The $b$-cycles $b_{j}, j=1, \ldots, g$, all start at the cut $C_{0}$, extend on one sheet to the cut $C_{j}$ and then return on the other sheet to $C_{0}$. Thus, $b_{j}$ intersects only the cycle $a_{j}$ and the intersection number $a_{j} \circ b_{j}$ is 1 , when oriented in the indicated way. However, in the computation of the periods in the following section, instead of dealing with these cycles, we make use of the contours $c_{j}, j=1, \ldots, g$ which link successive cuts as indicated in Fig. 2, Then one has the relation

$$
b_{j}=\sum_{k=1}^{j} c_{k}
$$

As mentioned in the introduction, the third option to use the code is to give the branch points in the form of a list of pairs. These pairs will be interpreted by 


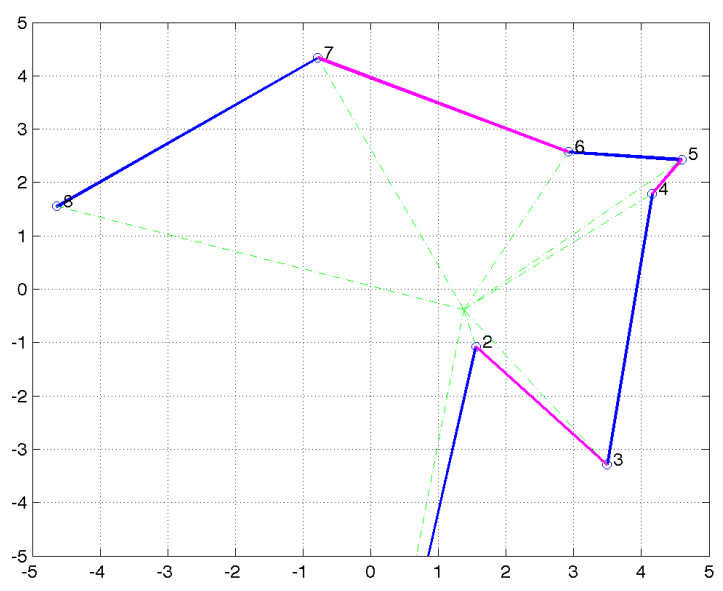

Figure 1. A generic situation. Six branch points chosen randomly are aligned using the argument with respect to their mean value. The blue lines indicate the cuts chosen on the basis of the sequence. The first branch point is at infinity.

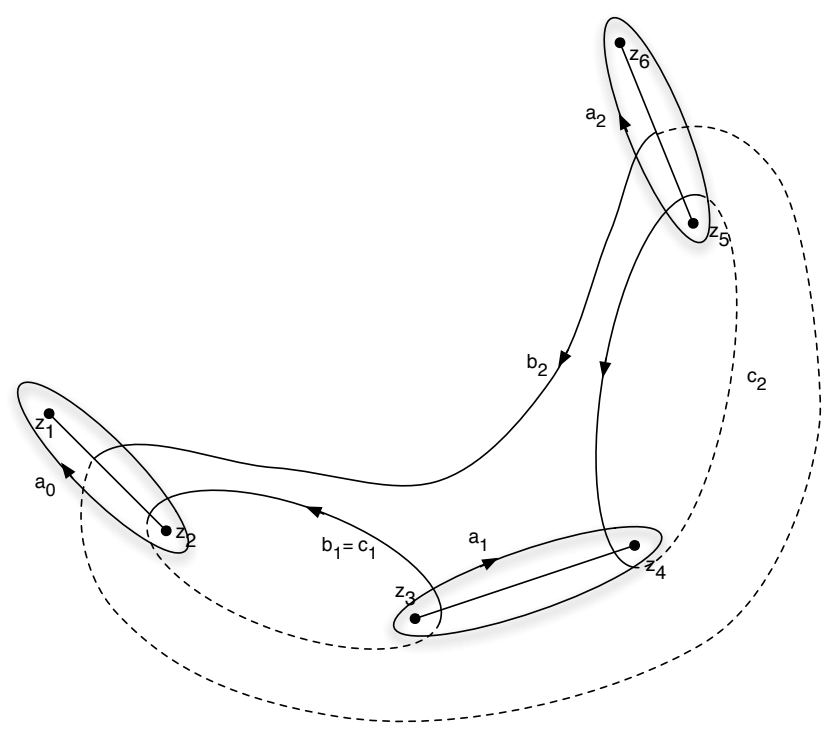

Figure 2. Basis of the homology used in the code

the code as corresponding to the cuts $C_{j}, j=0, \ldots, g$ in Fig. 2 in ascending order. Thus with the choice of these pairs of branch points, the system of cuts and thus the basis of the homology as in Fig. 2 are fixed. The cuts must be chosen in a way not to intersect other cuts, otherwise the code will produce an error.

\section{Computation of Abel map and periods of the Riemann surface}

In this section we compute the periods of the hyperelliptic surface, i.e., the integrals of the holomorphic one-forms along the cycles of the homology basis. A canonical basis $\omega_{k}, k=1, \ldots, g$ of holomorphic one-forms dual to a canonical basis 
of the homology can be obtained from the basis 2 by the normalization condition

$$
\int_{a_{j}} \omega_{k}=2 \pi \mathrm{i} \delta_{j k}, \quad j, k=1, \ldots, g .
$$

3.1. Analytic continuation of the root. To determine the periods of the surface, integrals of the form

$$
\int_{\Gamma} \nu_{k}=\int_{\Gamma} \frac{z^{k-1}}{\mu(z)} \mathrm{d} z
$$

for some contour $\Gamma$ on the hyperelliptic surface have to be computed. This requires the analytic continuation of the root $\mu(z)$ defined in eq. (1) along the contour. The general procedure for computing this function starts by parametrizing the contour $\Gamma$ by a real parameter $s \in[-1,1]$. For computational reasons, we choose a number $N_{c}+1$ of collocation points $s_{j}, j=0, \ldots, N_{c}$ on the contour. We sample the function

$$
\tilde{\mu}(z):=\sqrt{\prod_{i=1}^{N}\left(z-z_{i}\right)}, \quad \tilde{\mu}_{j}:=\tilde{\mu}\left(z\left(s_{j}\right)\right),
$$

on the collocation points obtaining a vector $\tilde{\mu}_{j}$, where we use the Matlab function sqrt to determine the square root. Note that this square root is branched along the negative real axis, whereas $\mu(z)$ by definition is an analytic function along the cuts defined in Fig. 1. Thus, in general, the function $\tilde{\mu}(z)$ as defined above will not be a smooth function on the contour $\Gamma$. To eliminate unwanted sign changes of $\tilde{\mu}(z)$, we determine the indices $j_{k}$ for which

$$
\left|\tilde{\mu}_{j+1}-\tilde{\mu}_{j}\right|>\left|\tilde{\mu}_{j+1}+\tilde{\mu}_{j}\right|
$$

i.e., the indices at which the Matlab root is branched along the contour $\Gamma$. This can be done in Matlab efficiently in a vectorized way, which means for all $j=1, \ldots, N_{c}$ at the same time. Then, for the $\tilde{\mu}_{j}$ with $j_{k} \leq j \leq j_{k+1}$ and $k$ odd, the sign of the $\tilde{\mu}_{j}$ is changed. The resulting function $\mu\left(z\left(s_{j}\right)\right)$ gives the analytic continuation of the root $\mu(z)$ along the contour $\Gamma$. Since a sign change and thus a quantity of order $2\left|\tilde{\mu}_{j}\right|$ has to be identified via $10 \mid$, the numerical result will be unique if the number $N_{c}$ of collocation points is sufficiently large to reliably distinguish $\tilde{\mu}_{j}$ and $\pm \tilde{\mu}_{j+1}$. This can be always achieved in practice as long as the branch points are sufficiently separated, i.e., if $\Delta$ in $(5)$ is smaller than $10^{14}$.

3.2. Computing the line integrals. To compute the periods of the holomorphic differentials $\nu_{k}$ in (2), the latter have to be integrated over the cycles $a_{j}$ and $b_{j}$ in Fig. 2. However, it is preferable to integrate directly on the cuts instead of closed contours around them in order to gain flexibility. This way, the relative position of the branch points can be essentially arbitrary (they just have to satisfy the condition $\Delta<10^{14}$ ) without the integration paths getting unnecessarily close to other branch points, which would affect numerical accuracy. In addition, the integration paths will always be the same no matter what the relative position of the branch points is. Thus, we consider the cycles in Fig. 2 in the limit that the cycle collapses to a contour touching the encircled respective cut on both sides,

$$
\lim _{\epsilon \rightarrow 0} \int_{C(\epsilon)} \nu_{k}
$$

where $C(\epsilon)$ is any contour which circles around two successive branch points, such as one of $a_{j}$ or $c_{j}$ and where $\epsilon$ is any measure of the transverse width of the contour across the line between the two branch points. We assume that in the limit $\epsilon \rightarrow 0$, this contour degenerates into two lines between the two branch points. Let us 
denote by $\Gamma_{i}^{ \pm}$the path along the line between $z_{i}$ and $z_{i+1}$ lying in the \pm -sheet of the Riemann surface, oriented from $z_{i}$ to $z_{i+1}$. Assuming the $a$-cycles to lie in the +-sheet, we have

$$
\lim _{\epsilon \rightarrow 0} a_{j}(\epsilon)=\Gamma_{2 j+1}^{+}-\Gamma_{2 j+1}^{-}, \quad \text { and } \quad \lim _{\epsilon \rightarrow 0} c_{j}(\epsilon)=\Gamma_{2 j}^{-}-\Gamma_{2 j}^{+} .
$$

Therefore, the contour integrals become

$$
\int_{a_{j}} \nu_{k}=2 \int_{\Gamma_{2 j+1}^{+}} \nu_{k}, \quad \int_{c_{j}} \nu_{k}=-2 \int_{\Gamma_{2 j}^{+}} \nu_{k} .
$$

Defining

$$
I_{j k}=2 \int_{z_{j}}^{z_{j+1}} \nu_{k}=2 \int_{\Gamma_{j}^{+}} \nu_{k}
$$

we have ( $I_{1 k}$ is the first cut, which does not contribute)

$$
A_{l k}=I_{2 l+1, k}, \quad B_{l k}=\sum_{j=1}^{l} I_{2 j, k} .
$$

So the problem to compute the periods of the holomorphic differentials $\nu_{k}$, $k=1, \ldots, g$ is reduced to the computation of some line integrals along a contour in the complex plane. Since the $\nu_{k}$ are holomorphic one-forms on the whole Riemann surface, they are in particular so on the contour. This makes a spectral approach attractive since it is well known that such approximations have exponential convergence for analytic functions.

We use here the Clenshaw-Curtis algorithm which is equivalent to an expansion of the integrand in terms of Chebyshev polynomials. As in the previous subsection, the contours are mapped to the interval $[-1,1]$ and a set of collocation points $s_{j}$, $j=0, \ldots, N_{c}$ is introduced on this interval. For Clenshaw-Curtis, the Chebyshev points $s_{j}=\cos \left(j \pi / N_{c}\right), j=0, \ldots, N_{c}$ are used. To compute an integral of the form $\int_{-1}^{1} f(s) \mathrm{d} s$, the function $f(s)$ is approximated by Chebyshev polynomials $T_{n}(s)$, $f(s) \approx \sum_{n=0}^{N_{c}} a_{n} T_{n}(s)$, where the coefficients $a_{n}$ are determined via a collocation method, i.e., by imposing equality of the previous relation at the collocation points $s_{j}, f\left(s_{j}\right)=\sum_{n=0}^{N_{c}} a_{n} T_{n}\left(s_{j}\right), j=0, \ldots, N_{c}$. Consequently, the integral of $f(s)$ is approximated by $\int_{-1}^{1} f(s) \mathrm{d} s \approx \sum_{n=0}^{N_{c}} a_{n} \int_{-1}^{1} T_{n}(s) \mathrm{d} s=\sum_{n=0}^{N_{c}} w_{n} f\left(s_{n}\right)$, where the $w_{n}$ are some known weights (depending on $N_{c}$ ) for the Clenshaw-Curtis method (see 33] and 34] for a Matlab code to compute the weights). Thus, the integration method consists of sampling the integrand on the Chebyshev collocation points and computing the scalar product of the vector of sampled values with the vector built from the weights $w_{n}$. As already mentioned, this method is known to show exponential convergence for analytic integrands, such as in the present case of the integration of holomorphic differentials on a hyperelliptic surface.

The periods are integrals of the form

$$
I=\int_{z_{1}}^{z_{2}} \frac{z^{k}}{\mu(z)} \mathrm{d} z
$$

where $\mu(z)$ is the analytically continued square root defined in the previous subsection. We compute this integral along the line between $z_{1}$ and $z_{2}$ which we parametrize by

$$
z:[-1,1] \rightarrow \mathbb{C}, s \mapsto z(s)=v+u s, \quad v:=\frac{z_{1}+z_{2}}{2}, \quad u:=\frac{z_{2}-z_{1}}{2} .
$$

Then $\mu(z)$ becomes

$$
\mu(z(s))=\sqrt{-u^{2}\left(1-s^{2}\right) p(z(s))},
$$


where $p(z)=\prod_{k \neq 1,2}\left(z-z_{k}\right)$. Thus the integral reads

$$
I=u \int_{-1}^{1} \frac{z(s)^{k}}{\sqrt{-u^{2}\left(1-s^{2}\right) p(z(s))}} \mathrm{d} s
$$

To regularize the integral we substitute $s=\sin \left(\frac{\pi}{2} t\right)$ with $\mathrm{d} s=\frac{\pi}{2} \cos \left(\frac{\pi}{2} t\right)$, and the integral becomes

$$
I=\frac{\pi}{2} \int_{-1}^{1} \frac{u \cos \left(\frac{\pi}{2} t\right)}{\sqrt{-u^{2} \cos ^{2}\left(\frac{\pi}{2} t\right) p(z(s(t)))}} z(s(t))^{k} \mathrm{~d} t= \pm \mathrm{i} \frac{\pi}{2} \int_{-1}^{1} \frac{1}{\sqrt{p(z(s(t)))}} z(s(t))^{k} \mathrm{~d} t .
$$

Since the decision about which sign to use will be made later we take here the positive sign. To evaluate this integral numerically we use the Clenshaw-Curtis algorithm as detailed above. The same procedure is applied for all integrals in (13) which gives the $(2 g+1) \times g$-matrix $I_{i k}$ of line integrals from which we can form the period matrices.

If one of the integration limits above is infinite, i.e., when $\infty$ is one of the branch points, then the procedure will be slightly altered. We apply a Möbius transformation to bring the infinite branch point to a finite location and then compute the appropriately transformed line integrals. In particular, we choose the inversion which interchanges $\infty$ with the central point $c: z \mapsto w=1 /(z-c)$. This works except for very symmetric cases when $c$ could be one of the branch points. Then we choose a random point for the inversion. The holomorphic differentials $\nu_{k}$ are transformed into

$$
\hat{\nu}_{k}=-\frac{(1+w c)^{k-1} w^{g-k}}{\tilde{\mu}(w)} \mathrm{d} w, \quad k=1, \ldots, g
$$

where $\tilde{\mu}(w)^{2}=w^{2 g+2} \mu(1 / w+c)^{2}$. After this transformation all branch points lie in a finite place and we compute the line integrals of the holomorphic differentials between infinity and the first branch point by computing the integrals of the transformed differentials $\hat{\nu}_{k}$ along the path between $c$ and the image of the first branch point. The other line integrals are computed as described above. Alternatively one could compute all the integrals with respect to the transformed variables, an approach which we have not explored so far.

Another possibility to address a branching of the surface at infinity is to use adapted local coordinates near infinity in the integration, i.e., $1 / \sqrt{z}$ as a local coordinate in an open neighborhood of infinity. To avoid numerical problems for $z \approx 0$, the integration path $\left[z_{j}, \infty\right]$ is split into two intervals $\left[z_{j}, R\right]$ and $[R, \infty]$ where $R$ is a point on the chosen ray towards infinity with $|R| \geq 1$ (we take $R$ to be the maximum of 1 and $2\left|z_{2}\right|$, the branch point of the cut extending to infinity). Thus an integral $J$ is computed as a sum of two integrals $J_{1}$ and $J_{2}$ on these two intervals. On the first interval, we use $s=\sqrt{z-z_{j}}$ as a local coordinate, and on the second we take $\tilde{s}=1 / \sqrt{z}$. In both cases we obtain an analytic integrand and use the Clenshaw-Curtis method as above to compute the integrals. The relative sign of these two integrals (recall that there is a sign ambiguity due to the square roots) is fixed by an auxiliary computation to a point $\tilde{z}$ on the integration path of the original integral with $\tilde{z} \gg R$. The result will not give an accurate approximation to the integral $J$, but will allow us to fix the sign in the sum $J_{1} \pm J_{2}$ uniquely. Both of the above approaches are implemented in the code and produce identical results within numerical accuracy.

3.3. Almost degenerate surfaces. An interesting limit of Riemann surfaces, for instance in the context of algebro-geometric solutions to integrable equations, is when the surface partially degenerates and the genus changes by one after pinching a cycle. In the case of a hyperelliptic surface, this means that two or more branch 
points coincide in the limit. In the case of a collapse of a branch cut, it is numerically convenient if the resulting double point on the curve is surrounded by an $a$-cycle (this facilitates the regularization procedure to obtain a Riemann surface of genus $g-1)$. Since the algorithm to arrange the branch points in subsection 2 does not take care of almost degenerate situations, as in 15 a third option to call the code exists where the branch points are prearranged to pairs representing the cuts encircled by the $a$-cycles by the user.

Since the cut-system in Fig. 2 is adapted to this case, the $a$-periods can be treated as before. For the $b$-periods, the fact that the branch points on the cut crossed by the $b$-cycle are close will affect numerical accuracy. For illustration, we consider the case that $z_{i} \approx z_{i+1}$. The corresponding $a$-period is computed as described in the previous subsection, and the integrand in (15) stays regular even in the limit $z_{i} \rightarrow z_{i+1}$. For the integral between $z_{i+1}$ and $z_{i+2}$ which is needed to compute the $b$-periods much higher numerical resolution would be needed since $z_{i} \approx z_{i+1}$. To address this problem, the integral from $z_{i+1}$ to $z_{i+2}$ is split into two integrals from $z_{i+1}$ to $\left(z_{i+1}+z_{i+2}\right) / 2$ and from $\left(z_{i+1}+z_{i+2}\right) / 2$ to $z_{i+2}$. In the former case we use for the integration the local coordinate

$$
z=\frac{z_{i}+z_{i+1}}{2}+\frac{z_{i+1}-z_{i}}{2} \cosh s, \quad s \in\left[0, \operatorname{arcosh}\left(\frac{z_{i+2}-z_{i}}{z_{i+1}-z_{i}}\right)\right]
$$

or

$$
s=\sqrt{z-z_{i}}
$$

if $z_{i}=\infty$ for the former integrand and

$$
z=\frac{z_{i+3}+z_{i+2}}{2}+\frac{z_{i+2}-z_{i+3}}{2} \cosh \tilde{s}, \quad \tilde{s} \in\left[0, \operatorname{arcosh}\left(\frac{z_{i+1}-z_{i+3}}{z_{i+2}-z_{i+3}}\right)\right]
$$

for the latter. In these local coordinates the integrands can be as well resolved numerically as the integrands of the $a$-periods even in situations close to the solitonic limit. After a linear transformation, the integrals are computed with the ClenshawCurtis routine. Again, the relative sign of these integrals has to be fixed because of the sign ambiguity in (15). This is done once more by directly computing the integral between $z_{i+1}$ and $z_{i+2}$ as in the case of the $a$-periods. This will not be as accurate as the result for the splitting of the integration path, but it will be enough to fix the relative sign.

Thus, the integrals will be computed efficiently with spectral accuracy, even if two branch points almost collapse. The sign ambiguity is the same as before and will be addressed in the following subsection. If more than two branch points almost collapse as for instance in the so-called positonic limit of $\mathrm{KdV}$, see [8] and references therein, the above procedure can only partially address the resulting numerical problems. As was shown in [8], it nonetheless still permits accurate numerical studies of the situation.

3.4. Fixing signs via Riemann's bilinear relations. Clearly, there is a sign ambiguity in the expression (15) on the right hand side. This can in principle be fixed by analytically continuing the square root as described in subsection 3.1 along a contour close to the contour given by the cuts in Fig. 1. But this is a numerically unstable procedure. Instead we always fix the sign only locally along any given cut (essentially accepting the choice made by Matlab), and we sort out the signs at the respective cuts a posteriori from the computed period matrices essentially using Riemann's bilinear identities. Since the cycle $a_{0}$ in Fig. 2 is homologous to a sum of the cycles $a_{j}, j=1, \ldots, g$, this implies a condition on the periods $A_{k l}$ in (14). This is a $(g+1) \times g$-matrix, which is the matrix of $a$-periods when we discard the first row. However, each column of this matrix must add up to zero if the root 
$\mu(z)$ is analytically continued according to Fig. 2. Since this is not enforced in our construction, we only know that there is a left null vector $n^{i}$, so that

$$
n^{i} A_{i k}=0 \text {. }
$$

If the function $\mu$ was analytically continued then this vector will be proportional to $(1,1, \ldots, 1)$, while there will be negative signs otherwise. In order to find the correct signs of the $n^{i}$, we identify the null space of $A_{i k}$. Matlab does this by performing a singular value decomposition (SVD) which is for an $m \times n$-matrix $M$ with complex entries given by $M=\mathcal{U} \Sigma \mathcal{V}^{\dagger}$; here $\mathcal{U}$ is an $m \times m$ unitary matrix, $\mathcal{V}^{\dagger}$ denotes the conjugate transpose of $\mathcal{V}$, an $n \times n$ unitary matrix, and the $m \times n$ matrix $\Sigma$ is diagonal (as defined for a rectangular matrix); the non-negative numbers on the diagonal of $\Sigma$ are called the singular values of $M$. The line in $\mathcal{U}$ corresponding to the singular value with smallest modulus gives the vector $n^{i}$. If sufficient numerical resolution is provided, i.e., if $N_{c}$ is large enough, this null space is 1-dimensional and can be normalized to be a vector with elements \pm 1 , i.e., $n^{i}= \pm 1$. A failure of this procedure implies that $N_{c}$ was too small, and that the code has to be rerun with a larger value of $N_{c}$ to compute the periods with higher precision. Again, the spectral convergence of the code is very useful here. Once the vector with components $n^{i}$ is correctly identified, it consists of \pm 1 's and we now replace $A_{i k} \leftarrow n^{i} A_{i k}$ (no summation over $i$ ) and hence fix the signs of the $a$-periods. The first row of $A_{j k}$ can then be discarded, and the resulting matrix is denoted by $\mathcal{A}$.

In order to fix the signs of the $b$-periods we proceed in a similar way. Note, that the real part of the Riemann matrix

$$
\mathbb{B}=2 \pi i \mathcal{B A}^{-1},
$$

where $\mathcal{B}$ is the matrix of $b$-periods of the holomorphic one-forms, must be symmetric and negative definite. The signs of those line integrals which make up the $a$-periods are already correct. Now we construct the $b$-periods according to (14). We allow for possibly different signs by writing

$$
B_{l k}=-\sum_{i=1}^{l} \epsilon_{i} I_{2 i, k}
$$

for $g$ undetermined signs $\epsilon_{i}$. Computing the matrix $\mathcal{B}$ and then the Riemann matrix with this expression, we can write down the symmetry condition on $\mathbb{B}$

$$
\operatorname{Re}\left(\mathbb{B}-\mathbb{B}^{t}\right)=0 \text {. }
$$

These are $\left(\begin{array}{l}g \\ 2\end{array}\right)$ conditions, which are linear in the $\epsilon_{i}$. We choose the $g-1$ conditions which come from the first off-diagonal of this matrix equation. This yields $g-1$ linear equations for the $g$ unknowns $\epsilon_{i}$. Thus, generically, we will again get a system with a 1-dimensional kernel which is identified as explained for the $a$-periods. This leads again to a null vector which has as entries only \pm 1 (up to numerical error of course) which give the relative signs of the line integrals to make the upper and lower off-diagonals in the real part of the Riemann matrix equal. We fix the remaining overall sign by the requirement that the real part of $\mathbb{B}$ should be negative definite. This completely fixes the signs of the line integrals.

The size of the remaining skew part of the real part of the Riemann matrix provides a good test of the accuracy of the numerical approach. We use the maximum of the $L^{\infty}$ norm of this skew part and the difference between the sum of the $a$ periods and the periods of the holomorphic one-forms along the cycle $a_{0}$ in Fig. 1 , denoted by err in the following, as an indicator of the accuracy of the computation. A typical example for this can be seen in Fig. 3. Visibly the error decreases exponentially with the number $N_{c}$ of Chebyshev points, i.e., we get the expected spectral convergence. This also holds in an almost degenerate situation as can be 
seen in the same figure, where pairs of branch points are separated only by $10^{-12}$ in the second example. In both cases machine precision is reached with $N_{c}=128$. It is remarkable that in the first case, $N_{c}=4$ already leads to an error of the order of a few percent. As mentioned, the code is very efficient. On an average computer, the computation of the periods takes of the order of $1 \mathrm{~ms}$ in the shown example for $N_{c}=128$. Note that the maximally achievable accuracy for the Riemann matrix in general depends on the conditioning (the range of the eigenvalues) of the matrix $\mathcal{A}$ of $a$-periods since the latter has to be inverted in $(16)$ to determine $\mathbb{B}$. This limits the achievable accuracy for the Riemann matrix for higher genus even if all periods are computed to the order of machine precision.

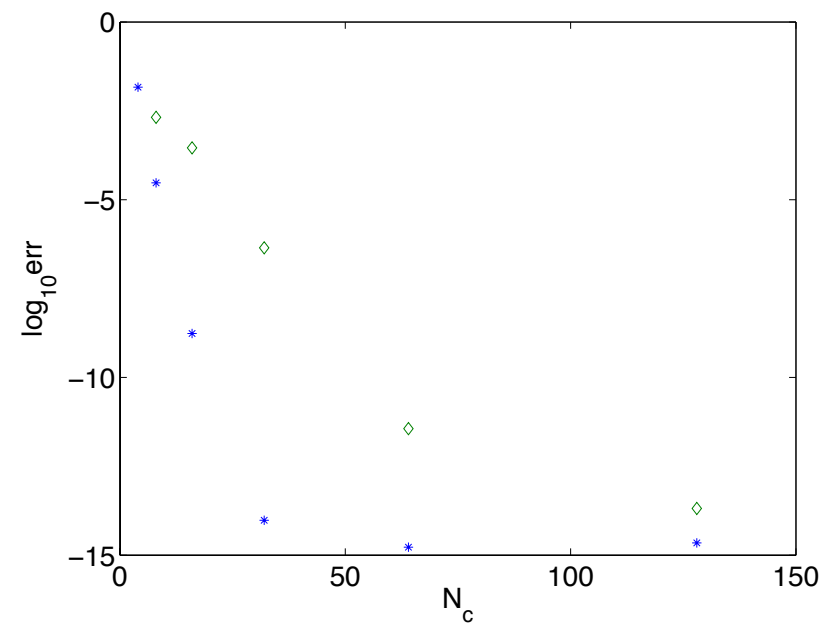

FiguRE 3. Numerical error err defined as the maximum of the $L^{\infty}$ norm of the skew part of the Riemann matrix and the $L^{\infty}$ norm of the sum of the $a$ periods including the period over $a_{0}$ in dependence of the number of Chebyshev points $N_{c}$ for the example of the genus 2 curve given by $\mu^{2}=z(z-1-\mathrm{i} \epsilon)(z-1+\mathrm{i} \epsilon)(z-2-$ $\mathrm{i} \epsilon)(z-2+\mathrm{i} \epsilon)$; the stars correspond to the case $\epsilon=1$, the diamonds to $\epsilon=10^{-12}$.

3.5. Abel map. Integrals of the holomorphic one-forms between arbitrary points $P, P_{0}$ of the hyperelliptic Riemann surface $\mathcal{R}$ can be computed essentially in the same way as the periods of the holomorphic differentials above. The Abel map

$$
P \mapsto \int_{P_{0}}^{P} \nu_{k}
$$

is a bijective map from the surface into the $\operatorname{Jacobian} \operatorname{Jac}(\mathcal{R})=\mathbb{C}^{g} / \Lambda$, where $\Lambda$ is the lattice formed by the periods of the holomorphic one-forms,

$$
\Lambda=\left\{2 \pi \mathrm{im}+\mathbb{B} \mathrm{n}: \mathrm{m}, \mathrm{n} \in \mathbb{Z}^{g}\right\} .
$$

To compute this map for a given point $P \in \mathcal{R}$, we identify the branch point closest to $P$, which will be denoted by $E$. Then we compute the integrals $\int_{E}^{P} \nu_{k}$ (we only discuss here the computation of the integral between $E$ and $P$, the one between $P_{0}$ and $E$ can then be computed in the same way). It is a consequence of the relations (14) that the Abel map between branch points on a hyperelliptic surface are half-periods. Thus, if $P$ is a branch point, the Abel map has been already computed above. If $P$ is a finite point, we use $s=\sqrt{z-E}$ as a local coordinate 
and compute the line integral after the analytic continuation of the square root as in 3.1 with the Clenshaw-Curtis algorithm applied to the integral in $s$.

If $P$ is a point covering infinity on a surface without branching at infinity, we introduce some intermediate point $Q$ with $\overline{Q E} \gg 1$. The integral to $Q$ is computed as described for a finite $P$. For the integral between $Q$ and $P$, we use $s=1 / z$ as a local parameter and compute the resulting integral again with the Clenshaw-Curtis method.

Since the Abel map is only defined up to periods of the holomorphic one-forms, we always choose it to be in the fundamental domain given by

$$
2 \pi \mathrm{i} \mathrm{p}+\mathbb{B} \mathrm{q}, \quad \mathrm{p}, \mathrm{q} \in \mathbb{R}^{g},
$$

with $-1 / 2<p_{i} \leq 1 / 2,-1 / 2<q_{i} \leq 1 / 2, i=1, \ldots, g$. In other words, the Abel map and thus an arbitrary point of the Jacobian can be given in terms of the $\left(p_{i}, q_{i}\right)$, which are called characteristics. Of special importance are half-integer characteristics with $2 \mathrm{p}, 2 \mathrm{q} \in \mathbb{Z}^{g}$. A half-integer characteristic is called even if $4\langle\mathrm{p}, \mathrm{q}\rangle=0 \bmod 2$ and odd otherwise. Here $\langle\cdot, \cdot\rangle$ denotes the Euclidean scalar product $\langle\mathrm{p}, \mathrm{q}\rangle=\sum_{i=1}^{g} p_{i} q_{i}$. The Abel map between branch points corresponds thus to a half integer characteristic.

\section{Sine-Gordon equation}

In this section we discuss solutions to the SG equation (4) in terms of multidimensional theta functions on hyperelliptic Riemann surfaces which were first constructed by Kozel and Kotlyarov [27, see also [1] and 30] for an alternative derivation based on Fay's trisecant identity. We present examples for real, smooth solutions, also in the solitonic limit. The solutions are given on Riemann surfaces with cuts between real points and/or cuts between complex conjugate points. Contrary to the previous code, the new version can handle surfaces with both types of cuts on the same surface which will be illustrated in this section. Note that the SG equation can be written in the form of a $1+1$ non-linear wave equation

$$
\phi_{t t}-\phi_{x x}=\sin (\phi), \quad \xi=\frac{x+t}{4}, \eta=\frac{t-x}{4} .
$$

We will discuss the solutions here only in dependence of the characteristic coordinates $\xi$ and $\eta$.

4.1. Solutions in terms of multi-dimensional theta functions. Solutions to the SG equation are given in terms of multi-dimensional theta functions which we define as an infinite series,

$$
\Theta_{\mathrm{pq}}(\mathrm{z}, \mathbb{B})=\sum_{\mathrm{N} \in \mathbb{Z}^{g}} \exp \left\{\frac{1}{2}\langle\mathbb{B}(\mathrm{N}+\mathrm{p}), \mathrm{N}+\mathrm{p}\rangle+\langle\mathrm{z}+\mathrm{q}, \mathrm{N}+\mathrm{p}\rangle\right\},
$$

with $\mathrm{z} \in \mathbb{C}^{g}$ and $\mathrm{p}, \mathrm{q} \in \mathbb{R}^{g}$ the characteristics.

The properties of the Riemann matrix ensure that the series converges absolutely and that the theta function is an entire function on $\mathbb{C}^{g}$. A characteristics is called singular if the corresponding theta function vanishes identically. Theta functions with odd (even) characteristics are odd (even) functions of the argument $\mathrm{z}$. The theta function with characteristics is related to the Riemann theta function $\Theta$, the theta function with zero characteristics $\Theta:=\Theta_{00}$, via

$$
\Theta_{\mathrm{pq}}(\mathrm{z}, \mathbb{B})=\Theta(\mathrm{z}+\mathbb{B} \mathrm{p}+\mathrm{q}) \exp \left\{\frac{1}{2}\langle\mathbb{B} \mathrm{p}, \mathrm{p}\rangle+\langle\mathrm{p}, \mathrm{z}+\mathrm{q}\rangle\right\} .
$$

The theta function has the periodicity properties

$$
\Theta_{\mathrm{pq}}\left(\mathrm{z}+2 \pi \mathrm{i} e_{j}\right)=e^{2 \pi i p_{j}} \Theta_{\mathrm{pq}}(\mathrm{z}), \quad \Theta_{\mathrm{pq}}\left(\mathrm{z}+\mathbb{B} e_{j}\right)=e^{-\left(z_{j}+q_{j}\right)-\frac{1}{2} B_{j j}} \Theta_{\mathrm{pq}}(\mathrm{z}),
$$


where $e_{j}$ is a vector in $\mathbb{R}^{g}$ consisting of zeros except for a 1 in jth position. In the computation of the theta function, the series $(18)$ is approximated as a sum. The $\operatorname{argument} \mathrm{z}$ is always written as $\mathrm{z}=\mathrm{z}_{0}+2 \pi \mathrm{im}+\mathbb{B} \mathrm{n}$, where $z_{0}$ is in the fundamental domain of the Jacobian. The theta function is computed for the argument $z_{0}$, and the relations 20 then give the theta function for the argument z. For details of the computation, the reader is referred to $[15$ and 16 .

Solutions to the SG equation can be obtained on a hyperelliptic Riemann surface given by

$$
\mu^{2}=z \prod_{i=1}^{2 g}\left(z-z_{i}\right), \quad \Re z_{i}>0
$$

in the form

$$
u=2 \mathrm{i} \ln \frac{\Theta_{\mathrm{pq}}(\mathrm{V} \xi+\mathrm{W} \eta+\mathrm{i} \pi \delta)}{\Theta_{\mathrm{pq}}(V \xi+W \eta)},
$$

where $\mathrm{V}, \mathrm{W} \in \mathbb{C}^{g}$ with components

$$
V_{j}=2 c_{j 1}, \quad W_{j}=\frac{2 c_{j g}}{\sqrt{\sum_{i=1}^{2 g} z_{i}}}, \quad j=1, \ldots, g,
$$

with $c_{i k}=2 \pi \mathrm{i}\left(\mathcal{A}^{-1}\right)_{i k}, i, k=1, \ldots, g$, where $\left[\begin{array}{l}\mathrm{p} \\ \mathrm{q}\end{array}\right]$ is an arbitrary nonsingular characteristics, and where $\delta \in \mathbb{C}^{g}$ with components $\delta_{i}=1$ for $i=1, \ldots, g$.

The solutions are real and regular (without poles), see the discussion in [1] (note that a different homology basis is used there), if the hyperelliptic surface is real, i.e., if the branch points $z_{i}$ are real or pairwise complex conjugate, and if the characteristic satisfies certain reality conditions. Let $\tau$ be the antiholomorphic involution of this surface acting on each sheet as the complex conjugation. On such a surface it is possible to introduce a basis of the homology of the form shown in Fig. 2 satisfying $\tau a_{j}=-a_{j}, j=1, \ldots, g$ and $\tau b_{j}=b_{j}$ if $b_{j}$ crosses a cut of real branch points only, and $\tau b_{j}=b_{j}-a_{j}$ if $b_{j}$ passes through a cut between conjugate branch points, see 11. Note that we do not always use such a cut system in the following since we also want to consider cuts collapsing away from the real axis. For the examples we will study below, we will always give the used characteristics and the branch points in pairs corresponding to the cuts encircled by the $a$-cycles.

Note that the solution 22 is only defined up to multiples of $2 \pi$. Since the logarithm in Matlab is branched on the negative real axis, solutions computed according to formula 22 will in general have jumps. As for the analytic continuation of the square root discussed in the previous section, we construct an analytic solution by comparing $u(\xi, \eta)$ on neighboring computed points $\left|u\left(\xi_{j}, \eta\right)-u\left(\xi_{j+1}, \eta\right)\right|$ to $\left|u\left(\xi_{j}, \eta\right)-u\left(\xi_{j+1}, \eta\right) \pm 4 \pi\right|$. If the latter expression is smaller than the former, a factor of $\pm 4 \pi$ is added. The same procedure is repeated for the coordinate $\eta$ so that we end up with a smooth solution $u$.

The numerical accuracy of the solution is controlled in two ways. First we check for each computed point $(\xi, \eta)$ that the identity for theta functions obtained by entering (4) with the solution $u$ of (22),

$$
\begin{aligned}
& \frac{\partial_{V W} \Theta_{\mathrm{pq}}(\mathrm{V} \xi+\mathrm{W} \eta+\mathrm{i} \pi \delta)}{\Theta_{\mathrm{pq}}(\mathrm{V} \xi+\mathrm{W} \eta+\mathrm{i} \pi \delta)}-\frac{\partial_{V} \Theta_{\mathrm{pq}}(\mathrm{V} \xi+\mathrm{W} \eta+\mathrm{i} \pi \delta) \partial_{W} \Theta_{\mathrm{pq}}(\mathrm{V} \xi+\mathrm{W} \eta+\mathrm{i} \pi \delta)}{\Theta_{\mathrm{pq}}(\mathrm{V} \xi+\mathrm{W} \eta+\mathrm{i} \pi \delta)^{2}} \\
& -\frac{\partial_{V W} \Theta_{\mathrm{pq}}(\mathrm{V} \xi+\mathrm{W} \eta)}{\Theta_{\mathrm{pq}}(\mathrm{V} \xi+\mathrm{W} \eta)}+\frac{\partial_{V} \Theta_{\mathrm{pq}}(\mathrm{V} \xi+\mathrm{W} \eta) \partial_{W} \Theta_{\mathrm{pq}}(\mathrm{V} \xi+\mathrm{W} \eta)}{\Theta_{\mathrm{pq}}(\mathrm{V} \xi+\mathrm{W} \eta)^{2}}=\frac{1}{2 \mathrm{i}} \sin u
\end{aligned}
$$

where the derivative of a function $F(\mathrm{z})$ with respect to a vector $U$ is defined as $\partial_{U} F(\mathrm{z}):=\langle\nabla F(\mathrm{z}), U\rangle$. This identity for theta functions is not built into the code 
and thus provides a strong test. The code reports a warning if the identity is not satisfied to better than $10^{-12}$.

In addition, the solutions $u$ are computed on Chebyshev collocation points for $\xi$ and $\eta$. As in the Clenshaw-Curtis integration in the previous section, this can be used to approximate the computed solution via Chebyshev polynomials. Since the derivatives of the Chebyshev polynomials can be expressed linearly in terms of Chebyshev polynomials, a derivative acts on the space of polynomials via a differentiation matrix. With these standard Chebyshev differentiation matrices (see 33,34 ), the solution can be numerically differentiated. With the computed derivatives we check to which numerical precision the partial differential equation (PDE) is satisfied by the numerical solution. If sufficient resolution is provided, the residual of the equation obtained with Chebyshev differentiation is smaller than $10^{-6}$.

4.2. Examples. We first study examples on real surfaces of genus 2. The branch points are given in pairs corresponding to the chosen cuts. The first cut is always between $-\infty$ and 0 , the remaining cuts are freely chosen according to the reality conditions. We always use $N_{c}=128$ Chebyshev points unless otherwise noted.

On the surface with the real branch points

$$
\left[\begin{array}{ccc}
-\infty & 1 & 3 \\
0 & 1+\epsilon & 3+\epsilon
\end{array}\right]
$$

we use the characteristics $\left[\begin{array}{l}\mathrm{p} \\ \mathrm{q}\end{array}\right]=\frac{1}{2}\left[\begin{array}{cc}1 & 1 \\ 1 / 2 & 1 / 2\end{array}\right]$. As can be seen in Fig. 4 . one obtains for $\epsilon=1$ a quasiperiodic solution. For $\epsilon \rightarrow 0$, this solution becomes the 2-kink solution of the SG equation as can be seen in the same figure on the right. Thus the solution on the non-degenerate surface can be seen as an infinite train of such 2-kinks.
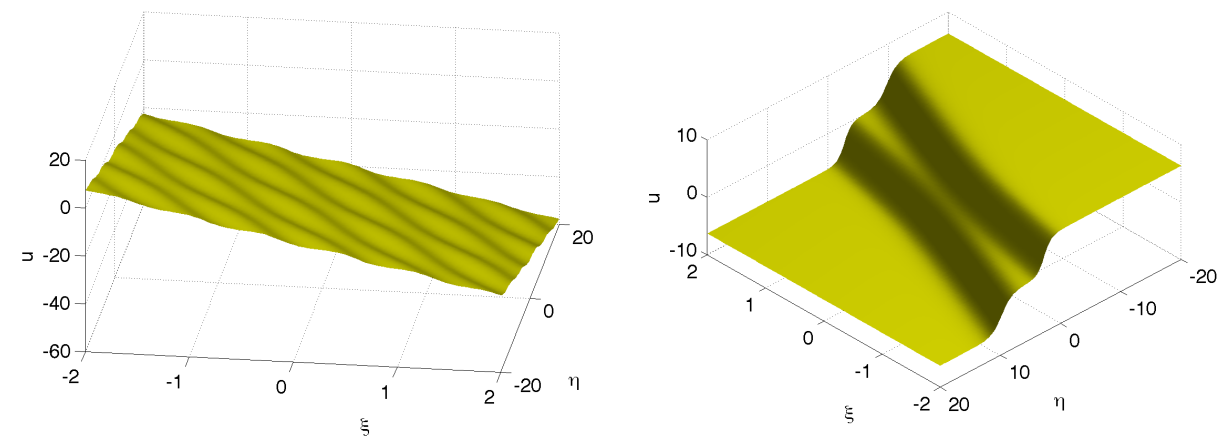

Figure 4. Solution 22 to the SG equation (4) on the surface of genus 2 with branch points $(23)$, on the left for $\epsilon=1$, on the right for $\epsilon=10^{-12}$.

On the surface with the cuts

$$
\left[\begin{array}{ccc}
-\infty & 1+\mathrm{i} & 1-\mathrm{i} \\
0 & 1+\mathrm{i}+\epsilon & 1-\mathrm{i}+\epsilon
\end{array}\right]
$$

i.e., only conjugate branch points except for $[-\infty, 0]$, we use the characteristics $\left[\begin{array}{l}\mathrm{p} \\ \mathrm{q}\end{array}\right]=\frac{1}{2}\left[\begin{array}{ll}1 & 1 \\ 0 & 0\end{array}\right]$. In the limit $\epsilon \rightarrow 0$, double points appear this time not on the real axis, but are conjugate to each other. The solutions for this case can be seen in 

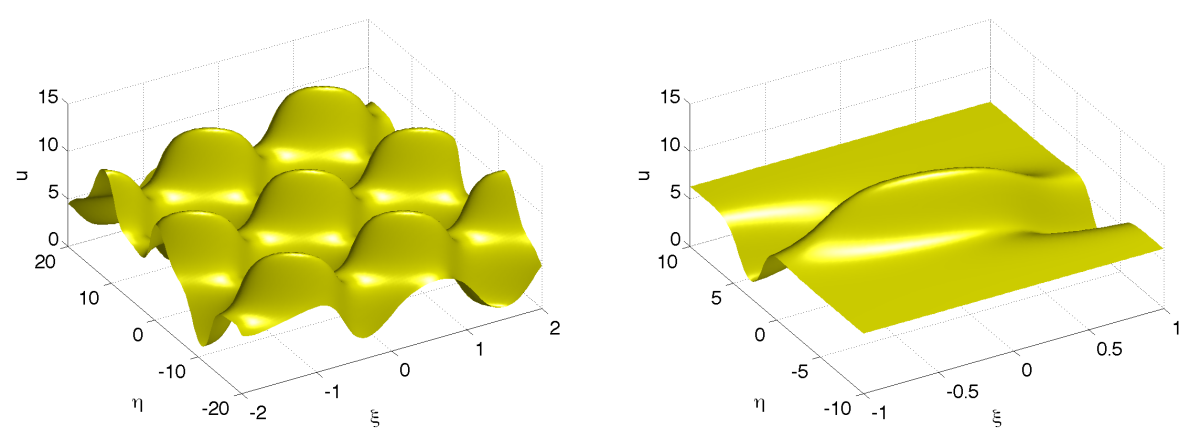

Figure 5. Solution (22) to the SG equation (4) on the surface with branch points (24); on the left for $\epsilon=1$, on the right for $\epsilon=10^{-12}$.

Fig. 5 for $\epsilon=1$ on the left and on an almost degenerate surface $\left(\epsilon=10^{-12}\right)$ on the right.

An example for mixed real and conjugate cuts in addition to the cut $[-\infty, 0]$ is given on the genus 2 surface with the cuts

$$
\left[\begin{array}{ccc}
-\infty & 1 & 3+\mathrm{i} \\
0 & 2 & 3-\mathrm{i}
\end{array}\right]
$$

for which we use the characteristics $\left[\begin{array}{l}\mathrm{p} \\ \mathrm{q}\end{array}\right]=\frac{1}{2}\left[\begin{array}{cc}1 & 1 \\ 1 / 2 & 1 / 2\end{array}\right]$. The corresponding solution can be seen in Fig. 6 .

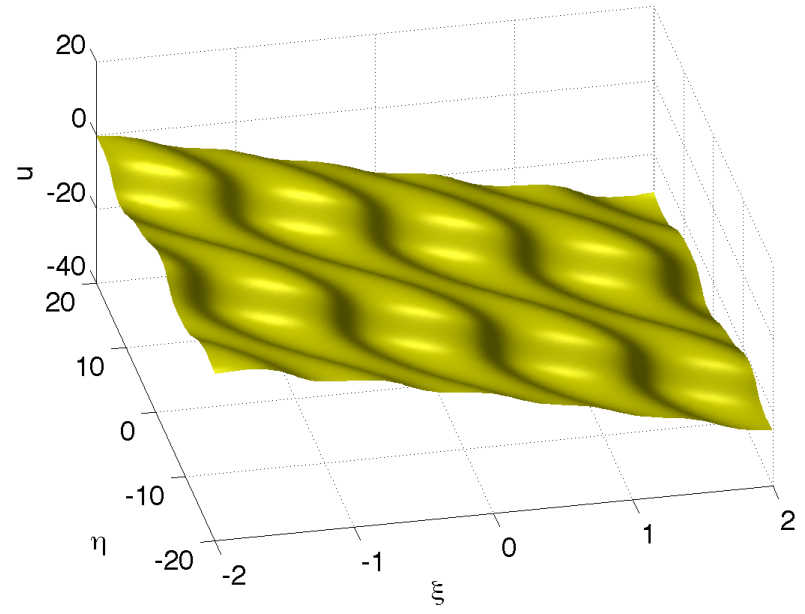

Figure 6. Solution (22) to the SG equation (4) on the surface with branch points $(25)$.

In Fig. 7, we show an example on a hyperelliptic Riemann surface of genus 4 with only real branch points,

$$
\left[\begin{array}{ccccc}
-\infty & 1 & 2 & 3 & 4 \\
0 & 1+\epsilon & 2+\epsilon & 3+\epsilon & 4+\epsilon
\end{array}\right]
$$


We use the characteristics $\left[\begin{array}{l}\mathrm{p} \\ \mathrm{q}\end{array}\right]=\frac{1}{2}\left[\begin{array}{cccc}1 & 1 & 1 & 1 \\ 1 / 2 & 1 / 2 & 1 / 2 & 1 / 2\end{array}\right]$. The 4-kink solution appearing in the solitonic limit on the right can be clearly recognized. The quasiperiodic solution on the non-degenerate surface can be seen as an infinite sequence of such kinks.
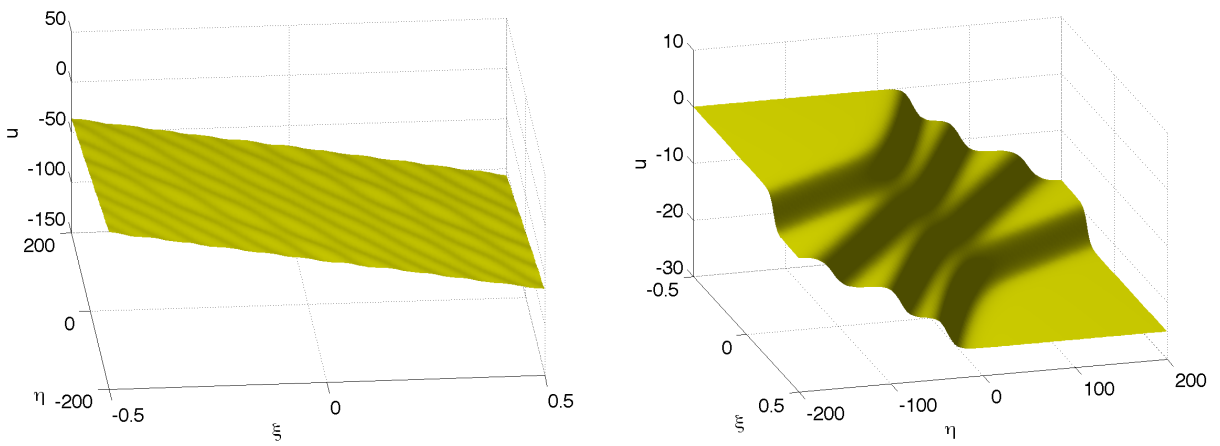

FIgURE 7. Solution (22) to the SG equation (4) on the surface with branch points $(26)$; on the left for $\epsilon=0.5$, on the right for $\epsilon=10^{-12}$.

A further example for a surface of genus 4 has the branch points,

$$
\left[\begin{array}{ccccc}
-\infty & 1+\mathrm{i} & 1-\mathrm{i} & 2+\mathrm{i} & 2-\mathrm{i} \\
0 & 1+\mathrm{i}+\epsilon & 1-\mathrm{i}+\epsilon & 2+\mathrm{i}+\epsilon & 2-\mathrm{i}+\epsilon
\end{array}\right]
$$

i.e., except for $[-\infty, 0]$ only conjugate cuts forming double points away from the real axis in the limit $\epsilon \rightarrow 0$. We use the characteristics $\left[\begin{array}{l}p \\ q\end{array}\right]=\frac{1}{2}\left[\begin{array}{llll}1 & 1 & 1 & 1 \\ 0 & 0 & 0 & 0\end{array}\right]$. To compute the case with $\epsilon=10^{-12}$, we use $N_{c}=512$ Chebyshev points.
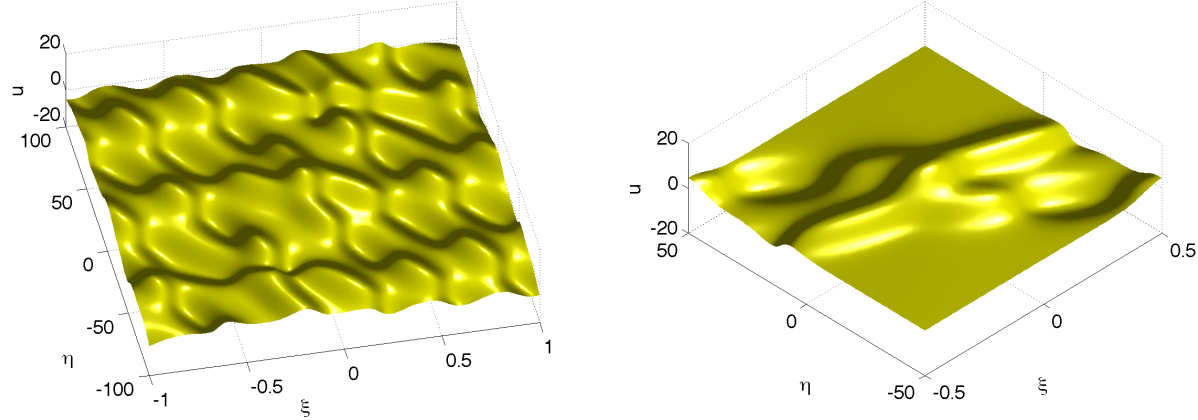

Figure 8. Solution (22) to the SG equation (4) on the surface with branch points (27); on the left for $\epsilon=0.5$, on the right for $\epsilon=10^{-12}$.

\section{OutLOOK}

In this paper, we have presented an algorithm to treat general hyperelliptic Riemann surfaces in Weierstrass form. The code is able to identify algorithmically a basis of the homology from a list of the branch points which was demonstrated for random points. It was shown that machine precision can be reached even in almost 
degenerate situations. This made it possible to study numerically the solitonic limit of solutions to the SG equation in terms of multi-dimensional theta functions.

The efficiency of the code allows the numerical study of functions on the modular space of hyperelliptic surfaces, see [32 for elliptic modular invariants. In [22], extremal properties of the determinant of the Laplacian in the Bergman metric on the modular space of genus 2 Riemann surfaces were considered. This determinant could be given, however, in terms of theta functions only. The present code would allow the study of similar questions for modular invariants involving integrals over the whole Riemann surface as Faltings' $\delta$-invariant $[12$ or the invariant studied in $[19$. This will be the subject of further research.

\section{REFERENCES}

[1] Belokolos, E.D., Bobenko, A.I., Enolskii, V.Z., Its, A.R., Matveev, V.B.: Algebro-geometric approach to nonlinear integrable equations. Springer, Berlin (1994)

[2] Bianchi, L.: Lezioni di Geometria Differenziale, (Pisa 1909)

[3] Bobenko, A.I., Klein, C. (ed.): Computational Approach to Riemann Surfaces, Lect. Notes Math. 2013 (2011)

[4] Bour E.: Théorie de la dformation des surfaces. J. Ecole Imperiale Polytechnique 19, 148 (1862)

[5] Deconinck, B., van Hoeij, M.: Computing Riemann matrices of algebraic curves. Physica D, 152153, 28 (2001)

[6] Deconinck, B., Heil, M., Bobenko, A., van Hoeij, M., Schmies, M.: Computing Riemann theta functions. Mathematics of Computation, 73, 1417-1442 (2004)

[7] Deconinck, B., and Patterson, M., in A.I. Bobenko, C. Klein, (ed.), Computational Approach to Riemann Surfaces, Lect. Notes Math. 2013 (2011).

[8] Dubard, P., Gaillard, P., Klein, C., Matveev, V.B.: On multi-rogue wave solutions of the NLS equation and positon solutions of the KdV equation, Eur. Phys. J. Special Topics Vol. $185,247258(2010)$

[9] Dubrovin, B.A.: Theta functions and non-linear equations, Usp. Mat. Nauk 36, No. 2, 11-80 (1981) (English translation: Russ. Math. Surv. 36, No. 2, 11-92 (1981)).

[10] Enolski, V.Z., Richter, P.H.: Periods of hyperelliptic integrals expressed in terms of $\theta$ constants by means of Thomae formulae, Phil. Trans. R. Soc. A 366 1005-1024 (2008)

[11] Faddeev LD, Korepin VE.: Quantum theory of solitons. Physics Reports 42 (1): 187 (1978)

[12] Faltings, G.: Calculus on Arithmetic Surfaces, Ann. Math. 119, 387-424 (1984)

[13] Frauendiener, J., Klein, C.: On the exact treatment of stationary counter-rotating dust disks: Physical Properties. Phys. Rev. D, 63, 84025 (2001)

[14] Frauendiener, J., Klein, C.: Hyperelliptic theta-functions and spectral methods. J. Comp. Appl. Math., 167, 193 (2004)

[15] Frauendiener, J., Klein, C.: Hyperelliptic theta-functions and spectral methods: KdV and KP solutions, Lett. Math. Phys., 76, 249-267 (2006)

[16] Frauendiener, J., Klein, C. in A.I. Bobenko, C. Klein, (ed.), Computational Approach to Riemann Surfaces, Lect. Notes Math. 2013 (2011)

[17] Grava, T., Klein, C.: Numerical solution of the small dispersion limit of Korteweg de Vries and Whitham equations, Comm. Pure Appl. Math., 60, 1623-1664 (2007)

[18] Grava, T., Tian, F.-R.: The generation, propagation, and extinction of multiphases in the KdV zero-dispersion limit. Comm. Pure Appl. Math., 55, no. 12, 1569-1639 (2002)

[19] de Jong, R.: Admissible constants for genus two curves. Bulletin of the LMS 42, 405411 (2010)

[20] Kalla, C., Klein, C.: New construction of algebro-geometric solutions to the Camassa-Holm equation and their numerical evaluation, Proc. Roy. Soc. A doi: 10.1098/rspa.2011.0583 (2012)

[21] Kamvissis, S., McLaughlin, K.D.T.-R. Miller, P.D., Semiclassical Soliton Ensembles for the Focusing Nonlinear Schrödinger Equation. Annals of Mathematics Studies, 154. Princeton University Press, Princeton, NJ (2003)

[22] Klein, C., Kokotov, A., Korotkin, D.: Extremal properties of the determinant of the Laplacian in the Bergman metric on the moduli space of genus two Riemann surfaces. Math. Zeitschr. 261(1), 73-108 (2009)

[23] Klein, C., Richter, O.: Ernst Equation and Riemann Surfaces. Lecture Notes in Physics 685 , Springer, Berlin (2005) 
[24] Korotkin. D.A.: Finite-gap solutions of the stationary axially symmetric Einstein equation in vacuum. Theor. Math. Phys. 77, 1018 (1989)

[25] Korotkin, D.: On some integrable cases in surface theory. J. Math. Sciences, 941177 (1999)

[26] Bobenko, A., Bordag, L.: Periodic multiphase solutions to the Kadomtsev-Petviashvili equation. J. Phys. A: Math. Gen., 22, 1259 (1989)

[27] Kozel, V.A., Kotlyarov, V.P.: Almost periodical solutions to the equation $U_{T T}-U_{X X}+$ $\sin U=0$, Kotlyarov, V.P.: Finite-gap solutions of the sine-Gordon equation, Dopovidi Akademii NAUK Ukrainskoi A-Fiz. Mat., 10, 878-881 (1976) English translation and summary of orginal papers at arXiv:1401.4410

[28] Kowalewski, S.: Sur le problème de la rotation d'un corps solide autour d'un point fixe, Acta Math., 14 pp. 8193 (1889)

[29] Lax, P.D., Levermore, C.D.: The small dispersion limit of the Korteweg de Vries equation, I,II,III. Comm. Pure Appl. Math., 36, 253-290, 571-593, 809-830 (1983)

[30] Mumford, D.: Tata lectures on theta. II, volume 29 of Progress in Mathematics. (Birkhäuser, Boston, MA, 1983)

[31] Neumann, C.: J. Reine Angew. Math., 56, 46-53 (1859)

[32] Quine, J.R., Sarnak, P. (ed): Extremal Riemann surfaces. Contemporary Mathematics, 201 AMS (1997)

[33] Trefethen, L.N.: Spectral Methods in Matlab. SIAM, Philadelphia, PA (2000)

[34] www.comlab.ox.ac.uk/oucl/work/nick.trefethen

[35] Zeng, Z.: Computing multiple roots of inexact polynomials. Math. Comp. 74, 869-903 (2004) E-mail address: joergf@maths.otago.ac.nz

Department of Mathematics and Statistics, University of Otago, P.O. Box 56, Dunedin 9010, NEW ZEALAND

E-mail address: Christian.Klein@u-bourgogne.fr

Institut de Mathématiques de Bourgogne, Université de Bourgogne, 9 avenue Alain Savary, 21078 Dijon Cedex, France 